ORIGINAL ARTICLE

\title{
Urinary tract infection: is there a need for routine renal ultrasonography?
}

\author{
G Zamir, W Sakran, Y Horowitz, A Koren, D Miron
}

Arch Dis Child 2004;89:466-468. doi: 10.1136/adc.2002.019182

\begin{abstract}
See end of article for authors' affiliations

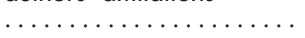

Correspondence to: Dr G Zamir, Pediatric Department $A^{\prime}$, Ha'Emek Medical Center, Afula, Israel; goorzamr@ netvision.net.il
\end{abstract}

Accepted 10 April 2003

\begin{abstract}
Aims: To assess the yield of routine renal ultrasound (RUS) in the management of young children hospitalised with first uncomplicated febrile urinary tract infection (UTI).

Methods: All children aged 0-5 years who had been hospitalised over a two year period with first uncomplicated febrile UTI in a medium size institutional regional medical centre were included. Children with known urinary abnormalities and/or who had been treated with antibacterial agents within seven days before admission were excluded. All included children underwent renal ultrasonography during hospitalisation and voiding cystouretrography (VCUG) within 2-6 months. The yield of RUS was measured by its ability to detect renal abnormalities, its sensitivity, specificity, and positive and negative predictive values for detecting vesicoureteral reflux (VUR), and by its impact on UTI management.

Results: Of 255 children that were included in the study, 33 children had mild to moderate renal pelvis dilatation on RUS suggesting VUR, of whom only nine had VUR on VCUG. On the other hand, in 36 children with VUR on VCUG the RUS was normal. The sensitivity, specificity, positive predictive value, and negative predictive value of abnormal RUS for detecting VUR were $17.7 \%, 87.6 \%, 23.5 \%$, and $83.2 \%$ respectively. In none of the patients with abnormal RUS was a change in the management at or following hospitalisation needed.

Conclusion: Results show that the yield of RUS to the management of children with first uncomplicated UTI is questionable.
\end{abstract}

$\mathrm{T}$ he main goals of imaging studies in children with a first episode of urinary tract infection (UTI) are to identify urinary tract anatomic abnormalities. If such abnormalities are found, therapeutic measures are executed in order to prevent future infections and possible long term damage to the kidneys. Currently, the recommended imaging study is renal ultrasound (RUS), which mainly detects abnormalities in the upper urinary tract such as hydronephrosis or obstruction, followed by voiding cystouretrography (VCUG) or radionuclide cystography (RNC) to detect anomalies of the lower urinary tract, mainly vesicoureteral reflux (VUR). ${ }^{12}$ The non-invasive nature, the lack of radiation, and the low cost of RUS have made it an ideal tool for the initial screening investigation in children with UTI. ${ }^{3}$ RUS, when performed during the hospitalisation may also detect pathologies such as obstructive uropathy or an abscess that directly influence the management of the child. However, these children usually have complicated course such as prolongation of fever.

Data have recently been published showing that RUS has a low sensitivity and specificity for predicting VUR. Also, many women might have undergone screening ultrasound examinations during pregnancy; major anomalies of the child's kidneys would therefore have already been known on admission. These data question the importance of routine RUS in the management children with acute uncomplicated UTI. ${ }^{5-8} 16$

The purpose of this study was to evaluate the yield of RUS to the management of young children with a first diagnosed episode of uncomplicated febrile UTI.

\section{PATIENTS AND METHODS}

This prospective study was conducted in Pediatric Departments $\mathrm{A}^{\prime}$ and $\mathrm{B}^{\prime}$ at Ha'emek Medical Center, Afula, Israel (a medium size regional hospital). The study population included all children under 5 years old who presented with first diagnosed uncomplicated febrile UTI between l January 1999 and 31 December 2000.
UTI was defined by the combination of a positive urine culture (growth of $\geqslant 100 \mathrm{bacteria} / \mathrm{ml}$ in a midstream sample or any growth in suprapubic bladder aspiration or in/out bladder catheterisation) and fever $\geqslant 38.0^{\circ} \mathrm{C}$.

Uncomplicated UTI was determined as a child with febrile UTI who clinically responded and became afebrile within 48 hours of initiation of therapy. Children with known urinary tract anomalies, and/or who had been treated with antibacterial agents within seven days before the admission were excluded. The study population size was calculated based on prevalence of VUR in $20 \%$ of young children with first diagnosed UTI. For power of 0.8 and significance $<0.05$, 200 children had to be included.

Urine cultures were obtained by suprapubic aspiration or in and out bladder catheterisation in children younger than 2 years and by the midstream techniques in older children. All children were initially treated with intravenous antibacterial agents (ampicillin + gentamicin or cefuroxime). Intravenous therapy was continued until fever had subsided, but for at least 96 hours in neonates or 48 hours in older infants and children. Thereafter, oral therapy with appropriate agents was continued for a total duration of 10-14 days. Preventive therapy was given thereafter until results of the VCUG were available.

RUS was performed in all patients during the hospitalisation using an ATL HDI 5000 with sector or linear 7 and 7.5 MHz transducers. It consisted of examination of the kidneys in order to show the kidney size, renal outlet obstruction (such as pelvic-uretro junction stenosis), collecting system dilatation, parenchymal structure, and parenchymal lesions such as an abscess. Also examination of the bladder was done in order to identify dilatation of the distal ureters, hypertrophy of the bladder wall, and presence of ureteroceles.

Abbreviations: DMSA, dimercaptosuccinic acid renal scan; RNC, radionuclide cystography; RUS, renal ultrasound; UTI, urinary tract infection; VCUG, voiding cystouretrography; VUR, vesicoureteral reflux 
Renal pelvis dilatation was defined as suggestive of VUR and graded as mild, moderate, or severe (hydronephrosis). ${ }^{9} \mathrm{~A}$ voiding cystourethrogram was performed within 2-6 months after the infection, and vesicoureteral reflux (VUR) was classified according to the international VUR classification. ${ }^{10}$ All imaging studies were read by experienced paediatric radiologists who were unaware of the study.

Impact on management was defined as a change of therapy, investigations, or follow up based on RUS results, that would not have been done otherwise.

Data were stored and analysed using Microsoft Excel. $\chi^{2}$ and $\chi^{2}$ for trend tests were used for comparison between specificity and sensitivity of different age groups. All $p$ values $<0.05$ were considered significant.

\section{RESULTS}

Overall during the study period 255 of 530 children met the inclusion criteria and were included in the study. Sixty three $(24.7 \%)$ were boys and $192(75.3 \%)$ girls; mean and median ages were 16 and 9 months respectively (range 8 days to 5 years). The median duration of fever in the hospital was 1 day (range 0-2 days). The median number of days from admission to RUS was 3 days (range 2-7). The main causative agents were Escherichia coli 229 (85\%), Klebsiella sp. 13 (5.1\%), and Proteus sp. 12 (4.7\%). Pseudomonas aeruginosa, Entrococcus fecalis, and Morganella morgani caused 11 infections.

Renal ultrasound showed urinary tract anatomical anomalies in three patients (1.2\%): one had an enlargement of the left kidney, one had a small renal cyst, and one had a unilateral double collecting system and severe hydronephrosis.

Abnormal RUS findings suggesting VUR were found in 33 (12.9\%) patients; these included mild unilateral pelvis dilatation in 32 patients and moderate unilateral pelvis dilatation in one patient.

Abnormal VCUG was found in $47(18.4 \%)$ patients. Thirteen patients had VUR grade 1, 18 patients had VUR grade 2, 13 patients had VUR grade 3, and one had VUR grade 4 (see table 1). One patient had ureterocele and right ectopic urethra and one had diverticuli in the vesicle.

Of the 33 patients whose RUS was suggestive of VUR, 26 patients $(78 \%)$ had normal VCUG, including the patient who had moderate unilateral dilatation of the collecting system. Seven patients $(21 \%)$ had VUR on VCUG: three had grade 1 , two had grade 2 , and two had grade 3 . Of the three patients with renal anomalies, the patient with a small cyst had a normal VCUG, the patient with enlargement of the left kidney had VUR grade 1 , and the patient with unilateral double collecting system and severe hydronephrosis had VUR grade 4.

Of 219 patients with normal RUS, 36 (16\%) had VUR on VCUG (see fig 1). RUS results did not cause a change in the management of any of the children during and following the admission, either in the antimicrobial agents or in the duration of intravenous therapy. The child who had a unilateral double collecting system was referred to further nephrological follow up. However, since he had VUR grade 4, he would have been referred anyway, so the RUS result did not change his management.

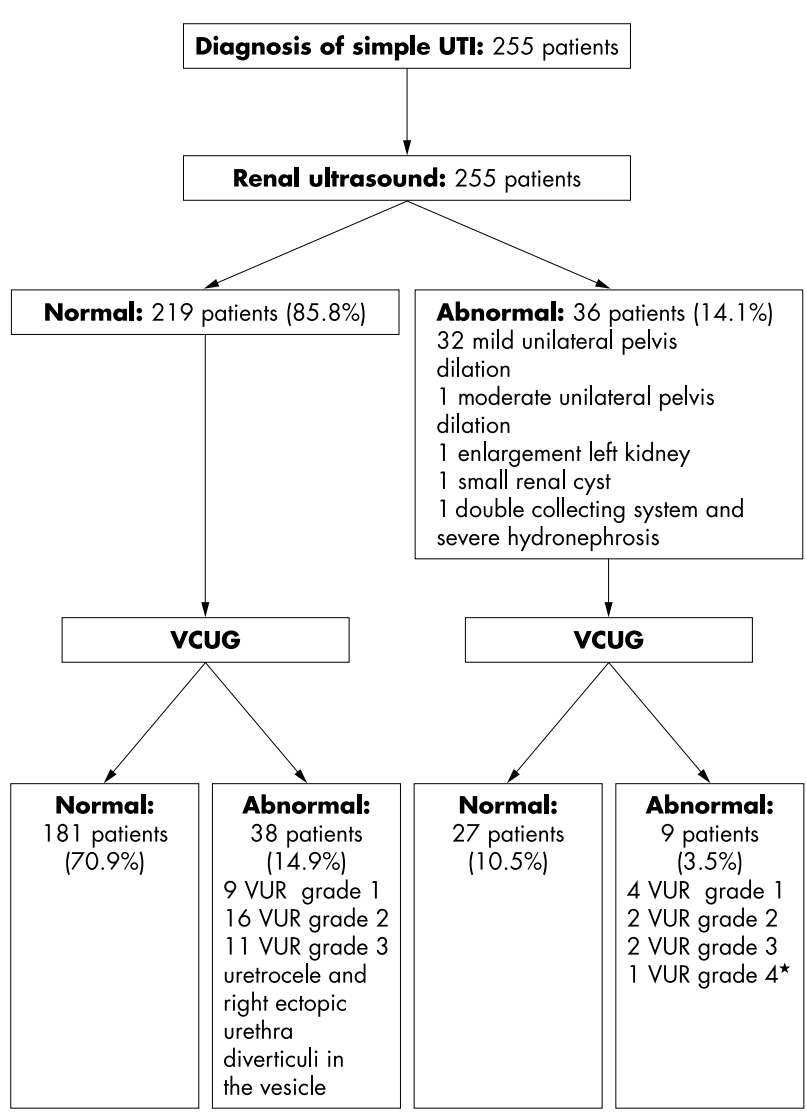

Figure 1 Results of imaging studies. *Also found to have double collecting system and severe hydronephrosis on RUS.

The sensitivity, specificity, positive predictive value, and negative predictive value of RUS for detecting VUR were $17.7 \%, 87.6 \%, 23.5 \%$, and $83.2 \%$ respectively (see table 2 ).

For the purpose of further analysis, the children were divided into three age groups: 0-2 months (71 children, 44/21 male/female), 2 months-2 years ( 122 children, 19/103 male/ female), and 2-5 years ( 62 children, $0 / 62$ male/female). The sensitivity, specificity, positive predictive value, and negative predictive value of abnormal RUS for detecting VUR were $27 \%, 80 \%, 20 \%$, and $86 \%$ respectively in the $0-2$ months group, $21 \%, 88 \%, 25 \%$, and $86 \%$ respectively in the 2 months-2 years group, and 6\%, 96\%, 33\%, and $76 \%$ respectively in the $2-5$ years group. The differences in sensitivity and specificity between these groups had no statistical significance.

\section{DISCUSSION}

Our study shows that RUS findings in children younger than 5 years admitted to hospital with a first episode of uncomplicated febrile UTI are of little value and have no influence on their management.

Table 1 Renal ultrasound findings in children with VUR on VCUG

\begin{tabular}{lccccc}
\hline \multirow{2}{*}{$\begin{array}{l}\text { RUS results } \\
\text { suggestive of VUR }\end{array}$} & \multicolumn{2}{l}{ VUR grade by VCUG } & \multicolumn{2}{l}{ Total } \\
\cline { 2 - 5 } & Grade 1 & Grade 2 & Grade 3 & Grade 4 & \\
\hline+ & $3(6.6 \%)$ & $2(4.4 \%)$ & $2(4.4 \%)$ & $1(2 \%)$ & $8(17 \%)$ \\
- & $10(22 \%)$ & $16(35 \%)$ & $11(24 \%)$ & $0(0 \%)$ & $37(82 \%)$ \\
Total & $13(29 \%)$ & $18(40)$ & $13(29 \%)$ & $1(2 \%)$ & $45(100 \%)$ \\
\hline
\end{tabular}


Table 2 The sensitivity, specificity, positive predictive value, and negative predictive value of RUS for detecting VUR by VCUG

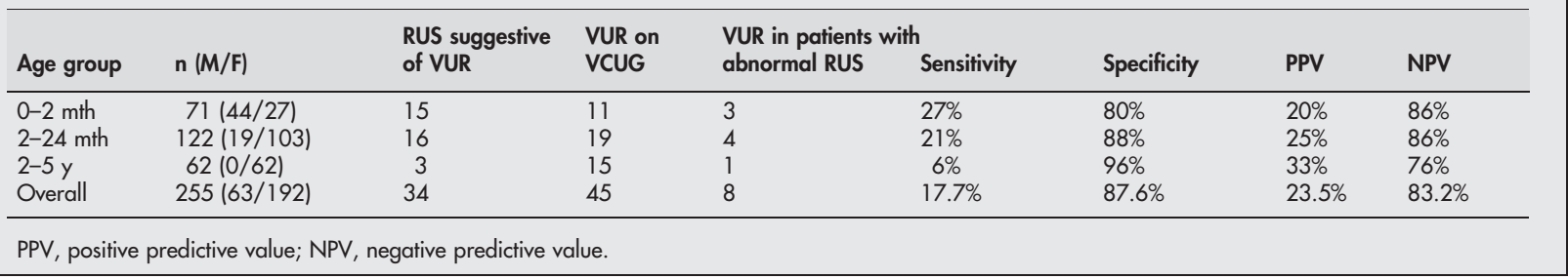

Several recent studies published have shown similar findings regarding the usefulness of RUS as a screening tool for VUR. Mahant and colleagues ${ }^{11}$ studied retrospectively 162 children under the age of 5 years with their first episode of UTI who had RUS and VCUG. RUS was suggestive of VUR if dilatation of the pelvi-calyces, dilatation of the ureters, or dilatation of the collecting system of one or both kidneys were reported. The overall prevalence of VUR was $22 \%$. RUS findings were suggestive of VUR in only 14 of 35 children with confirmed VUR, and in 30 of 127 children without VUR. The sensitivity, specificity, and positive and negative predictive values of ultrasound for VUR were $40 \%, 76 \%, 32 \%$, and $82 \%$ respectively.

Kass and colleagues ${ }^{12}$ evaluated 453 children with RUS, VCUG, and dimercaptosuccinic acid renal scan (DMSA). They showed that of 101 children who had a normal RUS and normal DMSA, 23\% had VUR using VCUG. Alon and Ganapathy $^{7}$ studied 124 patients with UTI, of whom RUS showed hydronephrosis and/or hydroureter in 10 patients (8.1\%); however, by VCUG, 38 patients (38\%) were found to have VUR. DiPietro and colleagues ${ }^{13}$ reported 70 children under the age of 5 years, who were studied using both RUS and VCUG. Five children (7\%) had abnormal RUS, of whom two had VUR on VCUG. Of the other 65 children with normal RUS, 19 (29\%) had VUR on VCUG. Smellie and Rigden ${ }^{14}$ evaluated four methods of investigation in 58 children following UTI. Thirty six patients $(62 \%)$ were found to have VUR by VCUG, but only eight (13\%) had abnormal RUS, giving a sensitivity, specificity, and false negative rate of $42 \%$, $91 \%$, and $78 \%$, of RUS for predicting VUR. She concluded that "ultrasonography is unreliable in detecting VUR, renal scarring, or inflammatory change and, alone, is inadequate for investigating UTI in children".

These studies show that RUS is an unreliable screening tool for VUR.

The contribution of RUS to the management of the hospitalised child with first simple UTI has been studied. Both Mucci and Maguire ${ }^{5}$ and Alon and Ganapathy ${ }^{7}$ found that routine RUS had a negligible effect on the clinical management of children with simple UTI. Our findings are in accordance with these results. In none of the children did the RUS finding change the management of the patients.

Goldman and colleagues ${ }^{15}$ reported similar findings in 45 neonates with UTI. Of 12 patients with abnormal RUS, four (33\%) had normal VCUG, while of 33 patients with normal RUS, $13(40 \%)$ had VUR on VCUG. However, he found urinary tract abnormalities in 22 of 45 (48\%) neonates compared to only $18 \%$ in our findings (13 of 71 ). This discrepancy in results can be partly explained by the patient selection methods, as Goldman et al included children who were suspected of urinary tract abnormalities by intrauterine ultrasound, while we excluded any child with known urinary tract anomalies.

In a recently published paper, Hoberman and colleagues ${ }^{16}$ studied 309 children, aged 1-24 months, using RUS, DMSA, and VCUG. They found that the sensitivity of RUS for detecting VUR on VCUG was $10 \%$, and PPV was $40 \%$. They also reported that the identified abnormalities did not modify management, and concluded that RUS and renal scanning at the time of the acute illness were of limited value. These results are generally in accordance with ours, and we concur with his conclusions.

Several issues still remain to be clarified: what is the role of intrauterine ultrasound, and does the imaging workup need to be changed according to its findings? Another point in question is the role of DMSA as a screening tool. Since we do not perform DMSA routinely we can not address the issue of VCUG versus DMSA based on our own data; however, in light of the growing amount of evidence against DMSA as a screening tool, further studies addressing this issue are needed.

In conclusion, both this and other studies question the yield of routine RUS in the management of young children with first simple UTI. We believe that RUS should only be performed in children in whom complications such as renal obstruction or abscess are suspected based on an unfavourable clinical course, or in children in whom VUR has been found, in order to look for renal structure abnormalities.

\section{Authors' affiliations}

G Zamir, Y Horowitz, D Miron, Pediatric Department A', Ha'Emek Medical Center, Afula, Israel

W Sakran, A Koren, Pediatric Department B', Ha'Emek Medical Center, Haifa, Israel

\section{REFERENCES}

1 American Academy of Pediatrics, Committee on Quality Improvement, Subcommittee on Urinary Tract Infection. Practice parameter: the diagnosis, treatment, and evaluation of the initial urinary tract infection in febrile infants and young children. Pediatrics 1999;103:843-52.

2 Jodal U, Lindberg U. Guidelines for management of children with urinary tract infection and vesico-ureteric reflux. Recommendations from a Swedish stateof-the-art conference. Swedish Medical Research Council. Acta Paediatr Suppl 1999;88:87-9.

3 Leoniads JC, McCauley RGK, Klauber GC. Sonography as a substitute for excretory urography in children with urinary tract infections. Am J Roentgenol 1984;144:815-19.

4 Mason WG. Urinary tract infection in children: renal ultrasound evaluation. Radiology 1984;153:109-11.

5 Mucci B, Maguire B. Does routine ultrasound have a role in the investigation of children with urinary tract infection? Clin Radiol 1994;49:324-5.

6 Hoberman A, Wald E. Urinary tract infections in young febrile children. Pediatr Infect Dis J 1997;16:11-17.

7 Alon US, Ganapathy S. Should renal ultrasonography be done routinely in children with first urinary tract infection? Clin Pediatr 1999;38:21-5

8 Alon US. More on urinary tract infection guidelines. Pediatrics 2001; 107:806.

9 Mittelstaedt CA, Vincent LM. Abdominal ultrasound. New York, NY: Churchill Livingstone, 1987:252.

10 Lebowitz RL, Olbing H, Parkkulainen KV, et al. International system of radiographic grading of vesicoureteric reflux. International Reflux Study in Children. Pediatr Radiol 1985;15:105-9.

11 Mahant S, Friedman J, MacArthur C. Renal ultrasound findings and vesicoureteral reflux in children hospitalized with urinary tract infection. Arch Dis Child 2002;86:419-20.

12 Kass EJ, Kernen KM, Carey JM. Paediatric urinary tract infection and the necessity of complete urological imaging. BJU Int 2000;86:94-6.

13 DiPietro MA, Blane CE, Zerin JM. Vesicoureteral reflux in older children: concordance of US and voiding cystourethrographic findings. Radiology 1997;205:821-2.

14 Smellie JM, Rigden SPA, Prescod NP. Urinary tract infection: a comparison of four methods of investigation. Arch Dis Child 1995;72:247-50.

15 Goldman M, Lahat E, Strauss GR, et al. Imaging after urinary tract infection in male neonates. Pediatrics 2000;105:1232-5.

16 Hoberman A, Charron M, Hickey RW, et al. Imaging studies after a first febrile urinary tract infection in young children. N Engl J Med 2003;348:195-202. 